\title{
Energy-efficient power allocation for massive M2M communication over LTE-A cellular uplink
}

\author{
Ning Li, Yingqing He*, Cong Wang, Yancheng Chen and Jianhui Xu
}

\begin{abstract}
In order to enable massive machine-to-machine (M2M) communication in Long Term Evolution-Advanced (LTE-A) cellular network, a machine-type communication gateway (MTCG) is introduced to solve the issue of control signaling overheads. In this paper, a power allocation algorithm based on the method of Lagrange multipliers is proposed for massive M2M communication over LTE-A cellular uplink. The proposed algorithm can decrease the signaling overheads by enabling the machine-type communication device (MTCD) to access the base station (BS) via a MTCG that collects the data from a group of M2M devices and forward it to the BS. The core objective of the proposed algorithm is to maximize the total energy efficiency of a group of M2M devices, while satisfying the time delay of the M2M devices, by coordinating the power transmission of the MTCDs and the MTCG. The simulation results demonstrate that the proposed algorithm outperforms the heuristic algorithm and has relatively close performance to the optimal design.
\end{abstract}

Keywords: Machine-to-machine communications, Power allocation, Long term evolution-advanced cellular network, Machine-type communication gateway, Machine-type communication device, Lagrange multipliers

\section{Introduction}

The number of radio devices that connect and transmit data to the radio network via Internet of Thing (IoT) [1] is increasing with the development of IoT. An important part of IoT is machine-to-machine (M2M) communication, also known as the machine-type communication (MTC). This type of communication refers to automatically connect the M2M devices, also called the machinetype communication devices (MTCDs), to the radio network without human intervenes [2, 3]. M2M communication is widely used in various applications such as remote data collection, information perception, remote vehicle scheduling, smart grit, E-health, and remote maintenance due to its special feature [4].

The Long Term Evolution-Advanced (LTE-A) cellular network plays an important role in the deployment of M2M communications due to its wide coverage and universal connectivity [5]. Enabling the M2M

\footnotetext{
*Correspondence: js_ningli@sina.com

${ }^{1}$ Institute of Communication Engineering, Army Engineering University of PLA, No. 88 Houbiaoying Street, 210007 Nanjing, China
}

communications in LTE-A cellular network can significantly expand the radio connectivity of MTCDs and promote the development of automation. Compared with the traditional human-to-human $(\mathrm{H} 2 \mathrm{H})$ communications, the M2M communications always consist of a large number of devices. The simultaneous communication of large number of MTCDs with the base station (BS) will result in network congestion and paralysis leading to communication failure and decrease in the quality of services (QoS) of $\mathrm{H} 2 \mathrm{H}$ communication, due to the limited radio resource $[6,7]$. In order to resolve this issue, a machine-type communication gateway (MTCG) is required in the LTE-A network for M2M communications. A MTCG can reduce the number of MTCDs that are directly connected to the $\mathrm{BS}$, decrease the control signaling overhead between the MTCDs and the BS, relieve the network congestions, and increase the spectral efficiency.

The MTCDs are always powered by a battery because they are usually deployed in dangerous or unreachable places. Therefore, the energy efficiency becomes a more serious matter for massive MTCDs than for the conventional $\mathrm{H} 2 \mathrm{H}$ user equipments $[8,9]$. Thus, the 
investigation of efficient radio resource allocation schedule and power control policy to increase the spectral efficiency while satisfying the time delay of MTCDs has become a trending research topic.

\subsection{Related works}

Limited literature is available about the power allocation for M2M communication between the BS and the MTCDs over the LTE-A cellular uplink. Ghavimi et al. [10] investigated the uplink scheduling and the power allocation for M2M communication in the LTE-A cellular network in order to improve the QoS, which considered the MTCD communication directly with the BS. They jointly allocated the sub-channel and the devices' transmit power to make maximize the sum-throughput and achieved improvement in the performance. However, they did not consider the energy efficiency of devices and when the massive MTCDs connect directly to the BS, it will rapidly increase the signaling overhead. Zhang et al. $[11,12]$ investigated the optimal power control for delay-constraint M2M communications over the cellular uplink and decreased the control signaling overhead by adding a MTCG to the communication link between the MTCDs and the BS. However, they only studied the power allocation and did not consider the power efficiency for the MTCDs. Aijaz et al. [13] went into energy-efficient uplink resource allocation with $\mathrm{M} 2 \mathrm{M} / \mathrm{H} 2 \mathrm{H}$ co-existence. They used the effective capacity as the throughput and considered the statistical time delay instead of the realtime delay of $\mathrm{M} 2 \mathrm{M} / \mathrm{H} 2 \mathrm{H}$ users. Multiple source power allocation with buffer-aided relay network using adaptive link selection has been investigated in [14]. However, this study did not consider the power allocation for the MTCDs and used the half-duplex transmission mode at the relay, considering only the statistical time delay. Tang et al. [15] [16] investigated the resource allocation to maximize the energy efficiency in MIMO broadcast channels and heterogeneous networks. However, they also did not consider the power allocation for relay MTCDs. All the abovementioned researches either focused on the energyefficient power allocation without signaling overhead or considered the power allocation without energy efficiency. Therefore, this paper investigates the energy efficient power allocation for massive M2M communications with real-time delay to decrease the signaling overhead.

\subsection{Contributions}

In this paper, the main focus is on the power allocation for massive M2M communication over the LTE-A cellular uplink. It introduces a MTCG that can collect data from a group of MTCDs and forward it to the BS in order to reduce the number of MTCDs that are directly connected to the BS, decrease the control signaling overhead between the MTCDs and the BS, and increase the spectral efficiency. The paper aims to maximize the total power efficiency of the MTCDs while satisfying the delay requirements of data transmission by jointly allocating the power to the MTCDs and the MTCG. The main contributions of this work are summarized as follows:

- The MTCG relay-based massive M2M uplink model is used to allocate the MTCDs and the MTCG's power. A MTCG can effectively collect the data from a group of MTCDs and forward it to the BS. Using the MTCG relay-based massive M2M uplink model, the control signaling overhead between the MTCDs and the BS is reduced and the spectral efficiency is increased.

- The total energy efficiency maximization for the MTCDs over the LTE-A cellular uplink is mainly focused in order to synergistically allocate power to the MTCDs and the MTCG. The framework is formulated as a total energy efficiency maximization problem, while respecting all the constraints associated with power allocation for the MTCDs and the MTCG.

- The total energy efficiency maximization problem that uses bits/joule as the energy efficiency metric is transformed into the total joules/bit energy efficiency minimization problem $[17,18]$. Then, a Lagrange multipliers-based power allocation algorithm [19] is proposed to solve the problem and obtain the sub-optimal power solution.

\subsection{Methods or experimental}

The remainder of this paper is organized as follows: Section 3 provides the MTCG relay-based massive M2M uplink model and discusses the problem formulation; Section 4 describes the proposed Lagrange multipliersbased power allocation algorithm. The numerical results and the conclusions are presented in Sections 5 and 6, respectively.

\section{System model and optimization problem}

\subsection{System model}

The cellular uplink is considered in a single cell of massive M2M devices over the LTE-A cellular network. As shown in Fig. 1, there are three communication types between MTCDs and the BS: (1) The MTCDs can directly exchange data bits with the BS by using the LTE-A specifications. (2) Apart from direct communication with the BS, the MTCDs can also communicate with the BS through a half-duplex MTCG. The link between the MTCG and the BS uses the LTE-A specifications, while the link between the MTCD and the MTCG can either use the LTE-A specifications or other personal area network (PAN), such as ZigBee and Bluetooth. (3) The MTCDs that are adjacent to each other can also communicate and exchange data bits. 


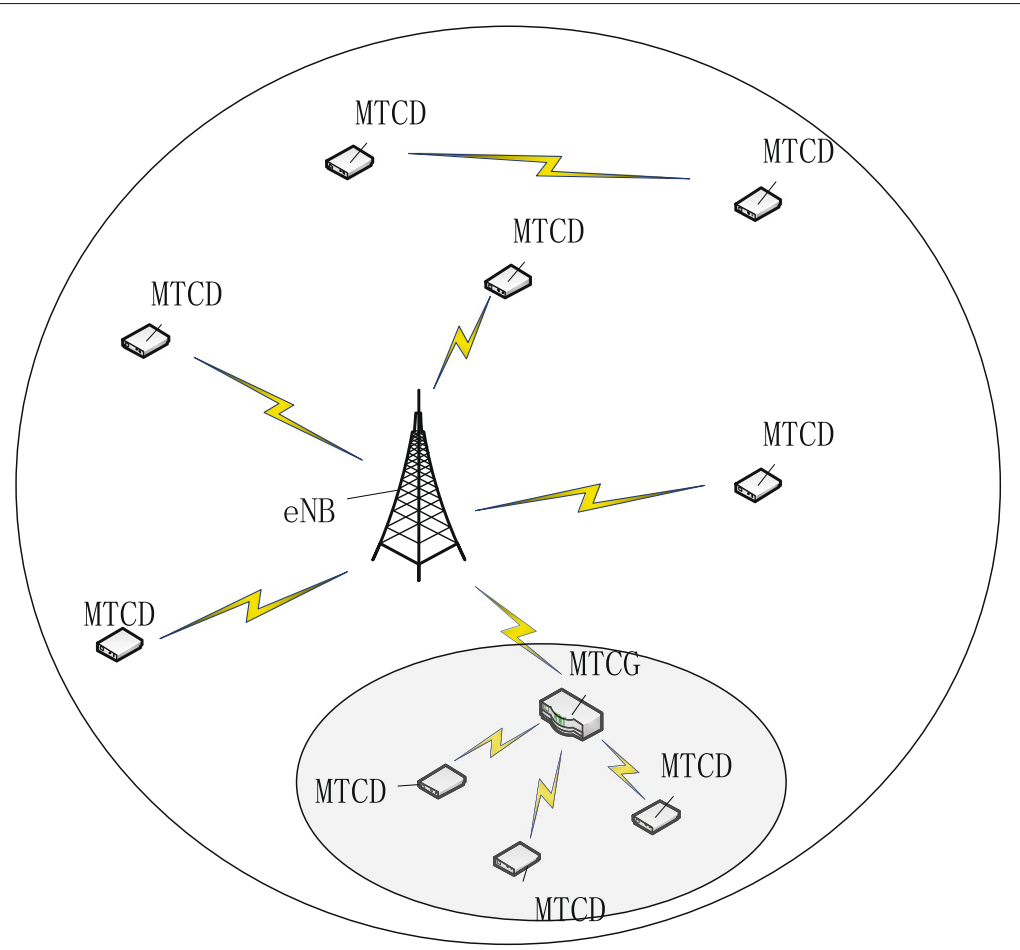

Fig. 1 The resource allocation model. It is assumed that the distance between the MTCDs and the BS is large enough to ensure that no M2M device communicates directly with the BS, and all the devices transmit data via the MTCG in the LTE-A uplink

This paper only considers the second communication type in which the MTCDs transmit data bits to the BS through the MTCG. The MTCDs are divided into several groups. Each MTCD of a group communicates with the $\mathrm{BS}$ via a MTCG that can connect $K$ active M2M users. It is assumed that $K=\{1,2, \cdots, K\}$. It is also assumed that the MTCG can simultaneously receive-and-forward information for the active MTCDs at the same frequency, which can be realized by using the full-duplex (FD) relay technology [20]. To this end, the MTCG should be equipped with two antennas, one for receiving the MTCDs' data and the other is for forwarding information to the BS. The use of two antennas will introduce self-interference to the MTCG; it is assumed that the severe self-interference from transmit antenna to receive antenna can be eliminated completely.

\subsection{Assumptions and LTE-A restrictions}

In the considered system model, it is assumed that both the MTCDs-to-MTCG and the MTCG-to-BS links use the LTE-A specifications. It is supposed that the M2M device $k$ needs to transmit $L_{k}$ bit data to the BS within time $T$, which can be treated as the time requirement that must be guaranteed from M2M device $k$ to the BS. The information of the MTCG connected with $K$ active MTCDs is $L_{G}=\sum_{k=1}^{K} L_{k}$, and the time for the MTCG-toBS link must be less than $T$, which is considered to be $T$ in this paper. The data of the MTCG will be less than $L_{G}$ in reality because it will use data aggregation [21] when the MTCG will be decoding or re-encoding the received data.

Assuming FD relay and receive-and-forward are used at the MTCG, the information transmission will be completed in two phases. In the first phase, the MTCDs transmit data bits to the MTCG that detects and decodes the received signals, while in the second phase, the MTCG re-encodes the received data and sent it to the BS. It will add time delays in the process of signal processing, but it is ignored in this work. Since the distances between the MTCDs and the BS are large enough, the MTCDs cannot directly communication with the BS.

There exist some restrictions in resource allocation while using the SC-FDMA in the LTE-A cellular uplink [22]. For the allocations of subcarriers: (1) exclusivity restriction, a single $\mathrm{RB}$ should only be allocated to at most one user; (2) adjacent restriction, multiple RBs allocated to a same user must be adjacent. For the allocation of power: (1) The peak power transmitted on a RB of a user must be less than a given peak power level $P_{G, l}^{\max }$; (2) The total power transmitted by user should not exclude the given total power level $P_{G}^{\max }$; (3) Power transmitted over all RBs that allocated to a same user must be equal. In this paper, the subcarrier allocation is not considered, so $P_{k}^{\max }$ and $P_{G}^{\max }$ are used as the maximal powers of the MTCDs $k$ and the MTCG, respectively. 


\subsection{Achievable data rates}

Assume that the channel gain between the MTCDs and the MTCG is the same as the channel coefficient between the MTCG and the BS; both are modeled as the frequency-flat and block-fading, i.e., the MTCG as well as each of the MTCDs experiences the same channel fading in one TTI. The channel coefficient $h_{m}$ is given by $h_{m}=$ $F_{m} \sqrt{1 / Z_{m}}$, where $F_{m}$ is the channel fading coefficient and $Z_{m}$ is the path loss [23]. The channel fading coefficient $F_{m}$ is always modeled as complex Gaussian random variable with zero mean and unit variance. The path loss $Z_{m}$ is related to the distance between the transmitter and the receiver. It is given by $Z_{m}=d^{\alpha}$, where $d$ is the distance and $\alpha$ is the path loss exponent, usually given from 2 to 6 , depending on the communication environment.

Then, the channel gains in each TTI from the M2M device $k$ to the MTCG and from the MTCG to the BS are $h_{k, G}=F_{k, G} \sqrt{1 / Z_{k, G}}$ and $h_{G, B}=F_{G, B} \sqrt{1 / Z_{G, B}}$, respectively. There will exist interference in the received signal for the MTCG from the transmitted signal due to incomplete elimination, $h_{S I}$ is used as the channel gain of self-interference link and $\Omega$ is used as the self-interference intensity.

By using Shannon's capacity formula, the upper bound on the achievable data rates (in bit/s) for the M2M device $k$ and the MTCG are respectively given by

$$
\begin{aligned}
& R_{k}=B_{k} \log _{2}\left(1+\frac{P_{k} h_{k, G}^{2}}{\sigma^{2}+P_{G} h_{S I}^{2}}\right), \\
& R_{G}=B_{G} \log _{2}\left(1+\frac{P_{G} h_{G, B}^{2}}{\sigma^{2}}\right) .
\end{aligned}
$$

where $B_{k}$ and $B_{G}$ are the transmit bandwidths of the M2M device $k$ and the MTCG, respectively, while $P_{k}$ and $P_{G}$ are the transmit powers of the M2M device $k$ and the MTCG, respectively, $\sigma^{2}$ is the power of Additive White Gaussian noise. The bandwidth of the MTCG is depended on the number of MTCDs connected to it.

\subsection{Optimization problem}

The required time for the M2M device $k$ to transmit $L_{k}$ data bits to the MTCG is given as $L_{k} / R_{k}$, and the MTCG requires $L_{G} / R_{G}$ time to forward $L_{G}$ data bits to the $\mathrm{BS}$. The energy efficiency is defined as the amount of data bits that can be transmitted per joule of energy consumed. Therefore, the power efficiency of each M2M device can be denoted as $\eta_{k}=\frac{L_{k}}{T P_{k}}$, and then, the sum-energy efficiency maximization power allocation problem can be derived as follows:

$$
\max \eta=\max \sum_{k=1}^{K} \eta_{k},
$$

subject to

$$
\begin{aligned}
& \text { a) } 0 \leq \mathrm{P}_{k} \leq P_{k}^{\max }, k=1,2, \ldots, K . \\
& \text { b) } 0 \leq \mathrm{P}_{G} \leq P_{G}^{\max } . \\
& \text { c) } \mathrm{L}_{k} / R_{k} \leq T, k=1,2, \ldots, K . \\
& \text { d) } \mathrm{L}_{G} / R_{G} \leq T .
\end{aligned}
$$

where $P_{k}^{\max }$ and $P_{G}^{\max }$ are the maximal transmitting powers of the M2M device $k$ and the MTCG, respectively. Constraint (a) and (b) are the transmit power restrains for the MTCDs and the MTCG, respectively, which means that the device's (MTCG's) power is positive and must be less than the maximal value. Constraint (c) and (d) are time requirements for the MTCDs to send data bits to the MTCG and for the MTCG to forward the received information to the BS, respectively. This implies that the transmission time from the MTCDs to the MTCG and from the MTCG to the BS both should not exceed $T$.

\section{The Lagrange multipliers-based power allocation algorithm}

Although the optimization problem (3) is more tractable to obtain the optimal solution, the calculation is quite large and requires complex computation to solve the problem directly, while the computing resources are limited for the system. Therefore, it is impractical to obtain the optimal solution directly from the optimal algorithm. To this end, a Lagrange multipliers-based power allocation algorithm is proposed that is relatively feasible in computational complexity.

In order to use the proposed algorithm, the total energy efficiency maximization problem (3) that uses bits/joule as the energy efficiency metric is transformed into the total joules/bit power efficiency minimization problem $[12,13]$ as follows:

$$
\min T \sum_{k=1}^{K} \frac{P_{k}}{L_{k}},
$$

subject to

$$
\begin{aligned}
& \text { a) } 0 \leq \mathrm{P}_{k} \leq P_{k}^{\max }, k=1,2, \ldots, K . \\
& \text { b) } 0 \leq \mathrm{P}_{G} \leq P_{G}^{\max } . \\
& \text { c) } \mathrm{L}_{k} / R_{k} \leq T, k=1,2, \ldots, K . \\
& \text { d) }) \mathrm{L}_{G} / R_{G} \leq T .
\end{aligned}
$$

Lemma 1 The solution of problem (3) is same as that for problem (4).

Proof Problem (3) and (4) are the inverse of each other, which can be decomposed into $\max \frac{L_{k}}{T P_{k}}$ and $\min \frac{T P_{k}}{L_{k}}$, respectively. When $P_{k}>0$, let $x=p_{k}$ and $a=\frac{L_{k}}{T}$, then the decomposition form of Problem (3) and (4) are $y_{1}=a \frac{1}{x}$ 
and $y_{2}=\frac{x}{a}$, respectively. The objective do is to prove that the solutions of $\max y_{1}$ and $\min y_{2}$ are the same. Now, taking the first-order derivatives of $y_{1}$ and $y_{2}$ will provide $y_{1}^{\prime}=-\frac{a}{x^{2}}<0, \forall x>0$, and $y_{2}^{\prime}=\frac{1}{a} x>0, \forall x>0$, respectively. This implies that $y_{1}$ and $y_{2}$ are monotonic subtraction and monotonic addition functions, respectively, and for any non-zero positive $x \in\left[x_{1}, x_{2}\right]$, the solutions of both $\max y_{1}$ and $\min y_{2}$ are at $x_{1}$. Hence, the solutions of $\max y_{1}$ and $\min y_{2}$ are the same, that is, the solution of problem (3) is same as that for problem (4).

In order to solve problem (4), the optimal values of this problem are obtained as the upper bounds of the proposed algorithm by using the build-in function "fmincon" in MATLAB. The "fmincon" function uses thee branch and bound method and the Liner programming relaxation to find the optimal solution. After that, the Lagrange multipliers-based power allocation algorithm is used to obtain the sub-optimal solution.

The Lagrange function of problem (4) is given as follows:

$$
\begin{aligned}
& \underset{P_{k}, P_{G} \geq 0, \gamma, \mu, \lambda, \rho \geq 0}{L\left(P_{k}, \boldsymbol{\gamma}, \boldsymbol{\mu}, \lambda, \rho\right)}=T \sum_{k=1}^{K} \frac{P_{k}}{L_{k}}-\sum_{k=1}^{K} \gamma_{k}\left(P_{k}^{\max }-P_{k}\right) \\
& \quad-\sum_{k=1}^{K} \mu_{k}\left(R_{k}-\frac{L_{k}}{T}\right)-\lambda\left(P_{G}^{\max }-P_{G}\right) \\
& \quad-\rho\left(R_{G}-\frac{L_{G}}{T}\right),
\end{aligned}
$$

subject to

$$
\begin{aligned}
& \text { a) } 0 \leq \mathrm{P}_{k} \leq P_{k}^{\max }, k=1,2, \ldots, K . \\
& \text { b) } 0 \leq \mathrm{P}_{G} \leq P_{G}^{\max } . \\
& \text { c) } \mathrm{L}_{k} / R_{k} \leq T, k=1,2, \ldots, K . \\
& \text { d) } \mathrm{L}_{G} / R_{G} \leq T .
\end{aligned}
$$

where $\boldsymbol{\gamma}=\left[\gamma_{1}, \gamma_{2}, \ldots, \gamma_{k}\right]^{T}$ and $\boldsymbol{\mu}=\left[\mu_{1}, \mu_{2}, \ldots, \mu_{k}\right]^{T}$ are the vectors of Lagrange multipliers for the MTCDs' transmit power and data rate constraints, respectively, while $\lambda$ and $\rho$ are the Lagrange multipliers for the MTCG's power and rate constraints, respectively.

In order to solve the problem (5), the first-order derivatives of $L\left(P_{k}, \boldsymbol{\gamma}, \boldsymbol{\mu}, \lambda, \rho\right)$ are obtained with respect to $P_{k}$ and $P_{G}$, respectively, and are made to be equal to zero. By substituting (1) and (2) into (5)

$$
\frac{\partial L}{\partial P_{k}}=\frac{T}{L_{k}}+\gamma_{k}-\mu_{k} \frac{B_{k} h_{k, G}^{2}}{\ln 2\left(\sigma^{2}+P_{G} h_{\mathrm{SI}}^{2}+P_{k} h_{k, G}^{2}\right)}=0,
$$

$$
\frac{\partial L}{\partial P_{G}}=\lambda-\rho_{k} \frac{B_{G} h_{G, B}^{2}}{\ln 2\left(\sigma^{2}+P_{G} h_{G, B}^{2}\right)}=0 .
$$

The sub-optimal transmit power of MTCDs and MTCG can be obtained by

$$
\begin{aligned}
& P_{k}^{*}=\frac{B_{k} \mu_{k} L_{k} h_{k, G}^{2}-\ln 2\left(T+L_{k} \gamma_{k}\right)\left(\sigma^{2}+P_{G} h_{S I}^{2}\right)}{\ln 2 h_{k, G}^{2}\left(T+L_{k} \gamma_{k}\right)}, \\
& P_{G}^{*}=\frac{B_{G} \rho h_{G, B}^{2}-\lambda \sigma^{2} \ln 2}{\lambda h_{G, B}^{2}} .
\end{aligned}
$$

After acquiring the solution of problem (5), the values of Lagrange multipliers $(\boldsymbol{\gamma}, \boldsymbol{\mu})$ and $(\lambda, \rho)$ are determined to obtain the solution of problem (4) by utilizing the ellipsoid and the subgradient methods [24], respectively (actually, the ellipsoid method is also a type of subgradient method; the only difference is that the former is used for a group of irrelevant variables while the latter is used for single variable). The Lagrange multipliers $(\boldsymbol{\gamma}, \boldsymbol{\mu})$ and $(\lambda, \rho)$ are jointly updated at each iteration, and problem (5) is solved to achieve the optimal $\left(\boldsymbol{\gamma}^{*}, \boldsymbol{\mu}^{*}\right)$ and $\left(\lambda^{*}, \rho^{*}\right)$. The subgradient of $L\left(P_{k}, \boldsymbol{\gamma}, \boldsymbol{\mu}, \lambda, \rho\right)$ is required by the ellipsoid method and the subgradient method at each iteration and can be readily given at $i$ th iteration by

$$
\begin{aligned}
& g_{i}\left(\gamma_{k}\right)=P_{k}^{\max }-P_{k}, k=1,2, \ldots, K, \\
& g_{i}\left(\mu_{k}\right)=R_{k}-\frac{L_{k}}{T}, k=1,2, \ldots, K, \\
& g_{i}(\lambda)=P_{G}^{\max }-P_{G}, \\
& g_{i}(\rho)=R_{G}-\frac{L_{G}}{T} .
\end{aligned}
$$

$\boldsymbol{g}_{i}=\left[g_{i}(\gamma), g_{i}(\mu)\right]^{T}$ is used as subgradients to update the multipliers $(\boldsymbol{\gamma}, \boldsymbol{\mu})$ at each iteration and the ellipsoid method to update the Lagrange multipliers $(\boldsymbol{\gamma}, \boldsymbol{\mu})$ as follows. First, the center of the ellipsoid is defined as $\boldsymbol{Z}=[\boldsymbol{\gamma}, \boldsymbol{\mu}]^{T}$, and the shape as $\boldsymbol{A}^{-1}$. Then, the ellipsoid is defined as:

$$
E\left(\boldsymbol{A}^{-1}, \boldsymbol{Z}\right)=\left\{\boldsymbol{x} \mid(\boldsymbol{x}-\boldsymbol{Z})^{T} \boldsymbol{A}^{-1}(\boldsymbol{x}-\boldsymbol{Z}) \leq 1\right\} .
$$

A minimal-volume new ellipsoid will be created at each iteration by using the subgradient that contains the other half of the past ellipsoid. Mathematically, the update algorithm is given as follows:

$$
\begin{aligned}
& \text { 1) } \tilde{g}_{i}=\frac{\boldsymbol{g}_{i}}{\sqrt{\boldsymbol{g}_{i}{ }^{T} \boldsymbol{A}_{i}^{-1} \boldsymbol{g}_{i}}}, \\
& \text { 2) } \boldsymbol{Z}_{i+1}=\boldsymbol{Z}_{i}-\frac{1}{N+1} \boldsymbol{A}_{i}^{-1} \tilde{\boldsymbol{g}}_{i} \text {, } \\
& \text { 3) } \boldsymbol{A}_{i+1}^{-1}=\frac{N^{2}}{N^{2}-1}\left(\boldsymbol{A}_{i}^{-1}-\frac{2}{N+1} \boldsymbol{A}_{i}^{-1} \tilde{\boldsymbol{g}}_{i} \tilde{\boldsymbol{g}}_{i}^{T} \boldsymbol{A}_{i}^{-1}\right) \text {. }
\end{aligned}
$$

where $N=2 K+M, K$ is the total number of MTCDs connected to the same MTCG, and $M$ is the variable used to match the update speed of the MTCDs' power with the MTCG's refresh rate. The initial value for shape $A^{-1}$ can be given as Eq. (22). 
The variables $(\lambda, \rho)$ are updated using the following simple iteration:

$$
\begin{aligned}
& \lambda_{i+1}=\lambda_{i}-\alpha_{i}^{\lambda} g_{i}(\lambda), \\
& \rho_{i+1}=\rho_{i}-\alpha_{i}^{\rho} g_{i}(\rho) .
\end{aligned}
$$

where $\alpha_{i}^{\lambda}$ and $\alpha_{i}^{\rho}$ are step sizes for updating $\lambda$ and $\rho$, respectively. Both do not depend on any data computed during the algorithm and must be determined before the algorithm is run [24]. Some basic step size rules are as follows: (1) Constant step size. In this case, the step size is a positive constant and is independent of iteration $i$. (2) Square summable but not summable. The step sizes satisfy $\alpha_{i} \geq 0, \sum_{i=1}^{\infty} \alpha_{i}^{2}<\infty, \sum_{i=1}^{\infty} \alpha_{i}=\infty$; one typical example is $\alpha_{i}=a /(b+i), a, b \geq 0$. (3) Nonsummable diminishing. The step sizes satisfy $\alpha_{i} \geq 0, \lim _{i \rightarrow \infty} \alpha_{i}=0, \sum_{i=1}^{\infty} \alpha_{i}=\infty$. The step sizes that satisfy this condition are called diminishing step size rules. A typical example is $\alpha_{i}=a / \sqrt{i}, a \geq$ 0 . In this paper, the square summable but not summable rule is used and the step sizes $\alpha_{i}^{\lambda}$ and $\alpha_{i}^{\rho}$ are given as:

$$
\begin{aligned}
& \alpha_{i}^{\lambda}=\frac{1}{C_{\lambda}+M+i}, \\
& \alpha_{i}^{\rho}=\frac{1}{C_{\rho}+M+i} .
\end{aligned}
$$

where $C_{\lambda}$ and $C_{\rho}$ are the constants for variables $\lambda$ and $\rho$, respectively, and $i$ is the iteration number order. The proposed Lagrange multipliers-based algorithm is summarized as Algorithm 1.

For the complexity analysis of the algorithm, the computation of $P_{G}^{*}, P_{k}^{*}$, and the update of the Lagrange multipliers $(\boldsymbol{\gamma}, \boldsymbol{\mu})$ and $(\lambda, \rho)$ are considered. In each iteration, it needs $K$ computations to calculate the MTCDs' power and 1 computation to calculate the MTCG's power, so the worst-case complexity is $O(I(K+1))$ with the algorithm executes to the maximum iteration $I$. However, in reality, it will not carry out to $I$. The ellipsoid method converges in $O\left((2 K)^{2}\right)$ iterations [25] with $2 K$ multipliers variable. Then, the worst-case complexity of the proposed algorithm is $O\left(I(K+1)+(2 K)^{2}\right)$, approximate to $O\left(I K+4 K^{2}\right)$.

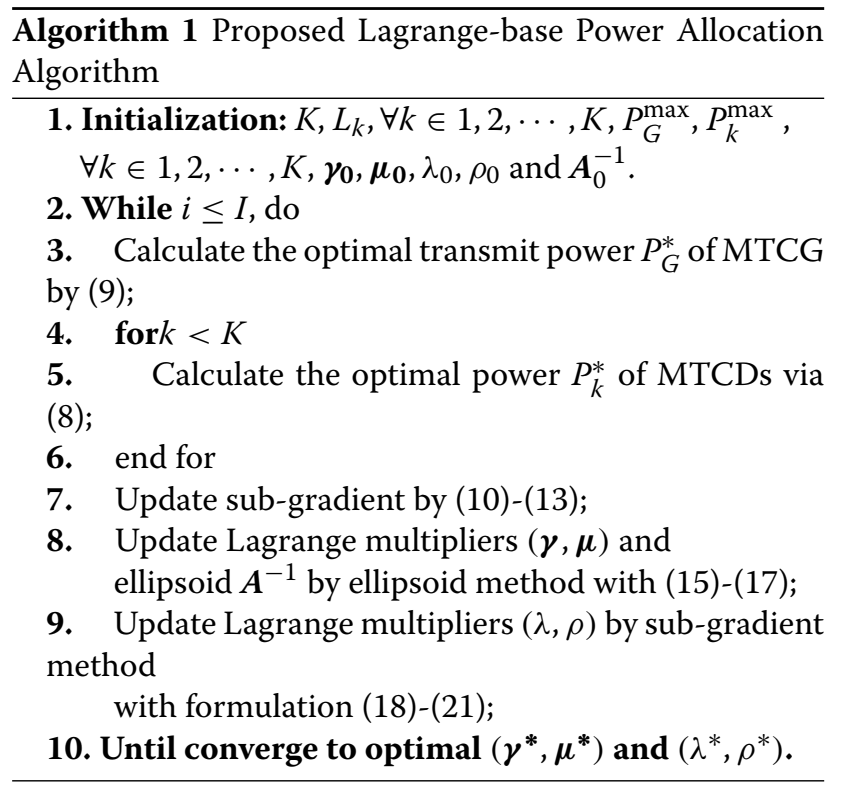

\section{Simulation results and discussions}

The simulation in this paper is based on the single cell, multi-users model in the LTE-A cellular uplink system. In the simulation, a MTCG and a group of $K=10$ MTCDs connected to the MTCG in the cell are considered. The distances from the MTCDs to the MTCG and the MTCG to the BS are randomly varied from 50 to 100 and 500 to $1000 \mathrm{~m}$, respectively. The channel fading accounts for small Rayleigh fading, large-scale path loss, and shadowing (log-normally distributed). The power of Additive White Gaussian noise is $\sigma^{2}=10^{-10}$, and the other simulation parameters are configured as $P_{k}^{\max }=14 \mathrm{dBm}$, $P_{\mathrm{G}}^{\max }=24 \mathrm{dBm}, T=1 \mathrm{~ms}, B_{k}=180 \mathrm{kHz}$, and $B_{G}=$ $B_{k}=1800 \mathrm{kHz}$.

In order to verify the feasibility of the proposed Lagrange multipliers-based power allocation algorithm, the solution for energy efficiency problem (4) is obtained

$$
\boldsymbol{A}_{0}^{-1}=\left(\begin{array}{cccccccccc}
N\left(\frac{\gamma_{1}}{2}\right)^{2} & 0 & \cdots & 0 & 0 & 0 & \cdots & 0 & 0 & 0 \\
0 & N\left(\frac{\gamma_{2}}{2}\right)^{2} & \cdots & 0 & 0 & 0 & \cdots & 0 & 0 & 0 \\
0 & 0 & \ddots & 0 & 0 & 0 & \cdots & 0 & 0 & 0 \\
\vdots & \vdots & \cdots & N\left(\frac{\gamma_{K}}{2}\right)^{2} & \vdots & \vdots & \ddots & \vdots & \vdots & \vdots \\
0 & 0 & \cdots & 0 & N\left(\frac{\mu_{1}}{2}\right)^{2} & 0 & \cdots & 0 & 0 & 0 \\
0 & 0 & \cdots & 0 & 0 & N\left(\frac{\mu_{2}}{2}\right)^{2} & \cdots & 0 & 0 & 0 \\
\vdots & \vdots & \ddots & \vdots & \vdots & \vdots & \ddots & \vdots & \vdots & \vdots \\
0 & 0 & \cdots & 0 & 0 & 0 & \cdots & N\left(\frac{\mu_{K}}{2}\right)^{2} & 0 & 0 \\
0 & 0 & \cdots & 0 & 0 & 0 & \cdots & 0 & N\left(\frac{\lambda}{2}\right)^{2} & 0 \\
0 & 0 & \cdots & 0 & 0 & 0 & \cdots & 0 & 0 & N\left(\frac{\rho}{2}\right)^{2}
\end{array}\right)
$$




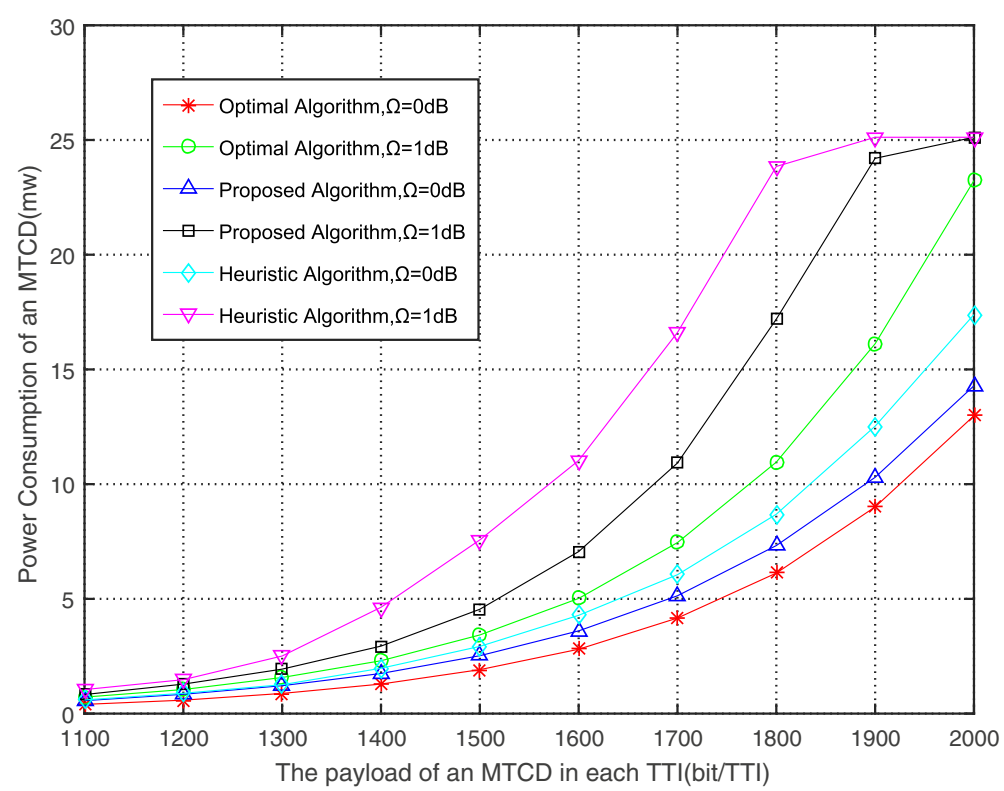

Fig. 2 The power consumption with different payloads. The MTCDs are uniformly distributed in 100-m radius of the MTCG, and the MTCG is uniformly distributed in 1000-m radius of the BS. The number of groups of MTCDs is $K=10$. The other configuration parameters are $P_{k}^{\max }=14 \mathrm{dBm}, P_{\mathrm{G}}^{\max }=24 \mathrm{dBm}, T=1 \mathrm{~ms}, B_{k}=180 \mathrm{kHz}$, and $B_{G}=K B_{k}=1800 \mathrm{kHz}$

first by using the built-in function "fmincon" in MAT$\mathrm{LAB}$, which is considered as the optimal algorithm and treated as the upper bound of the achievable performance. Both scenarios, MTCG with self-interference $(\Omega=1 \mathrm{~dB})$ and without self-interference $(\Omega=0 \mathrm{~dB})$, are considered. The data to be transmitted by the MTCDs in each TTI is changed from $1.1 \mathrm{Kbit} / \mathrm{TTI}$ to $2 \mathrm{Kbit} / \mathrm{TTI}$, and the performances of the optimal algorithm, the proposed Lagrange multipliers-based algorithm, and the heuristic algorithm [9] are compared. The heuristic algorithm's main process is as follows: firstly, the power of MTCDs and MTCG is computed based on the minimal data rate; then, the power of MTCDS and MTCG is updated to satisfy the restriction of SC-FDMA in reference's assumption; and lastly, the final power allocation is obtained by coordinating the MTCG's power.

Figure 2 represents the relationship between the power consumption and the data to be transmitted by the M2M devices. It can be seen from the figure that the power consumption of devices increases with the increase in the data to be transmitted in each TTI. The reason is that with the data increase, the devices must increase their transmit power to ensure that the real transmit time satisfies the time requirements. The power consumption of MTCDs varies rapidly when there is no self-interference from the transmit signal at the MTCG. This is due to the fact that the MTCDs need more transmission power to balance out the MTCG's self-interference and ensure essential decode performance for the MTCG when there is interference from the MTCG's transmit signal. It is obvious from Fig. 2 that the proposed Lagrange multipliersbased power allocation algorithm is much better than the heuristic algorithm in simulation performances, and the consumed power of each MTCD is closer to the optimal algorithm, with low power consumptions.

Figure 3 illustrates the relationship between the data bit to be transmitted by the MTCDs and the total bit/joule energy efficiency. The figure shows that the total power efficiency decreases with the increase of data bits to be transmitted for the MTCDs. The reason is that when the data increases, the devices' power also increases simultaneously. However, the growth rates of devices' transmit power are greater than the increase speed of the data bits, which results in the decrease of total power efficiency. It is apparent that the total energy efficiency of the MTCDs is higher when there is no self-interference at the MTCG than when there exists self-interference. Furthermore, it can be clearly seen that the total power efficiency of the proposed algorithm is better than that of the heuristic algorithm, and more approximates the optimal algorithm.

Figure 4 illustrates convergence behavior of the three algorithms over the number of iterations. The complexities of the three algorithms are summarized as follows: all the algorithms converge to stable energy efficiency when they run with number of iterations. The proposed Lagrange multipliers-based algorithm converges more quickly than the optimal algorithm but more slowly than the heuristic algorithm (For the optimal algorithm 


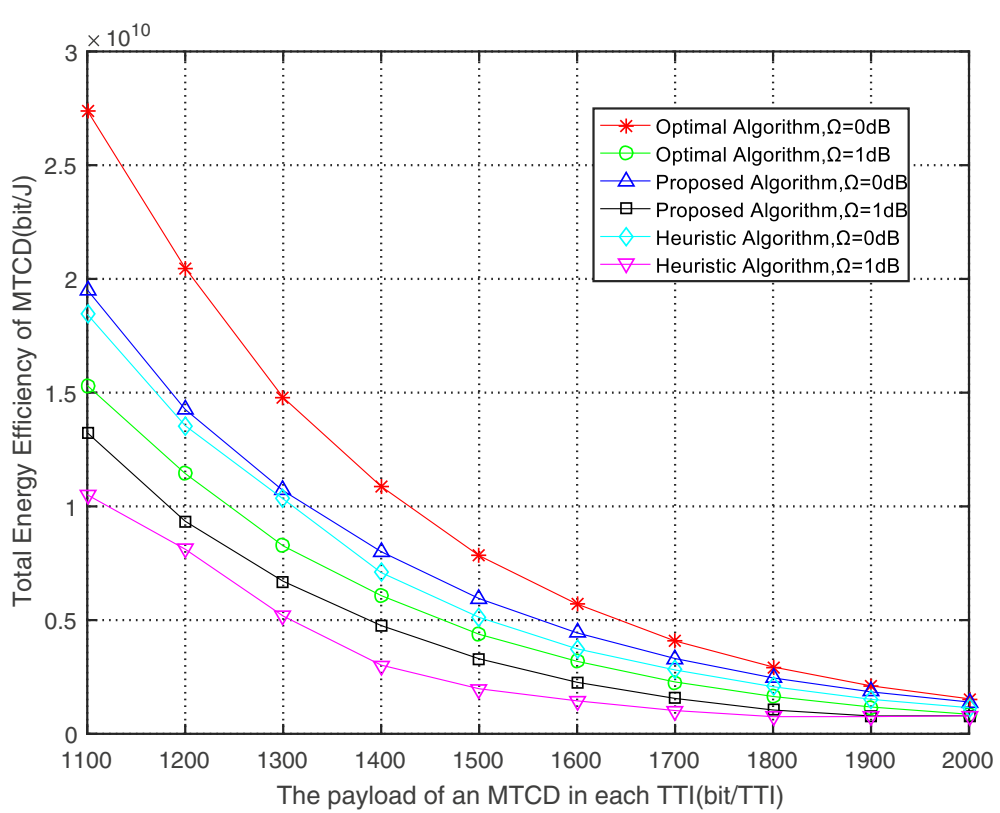

Fig. 3 The total energy efficiency with different payloads. The MTCDs are uniformly distributed in 100-m radius of the MTCG, and the MTCG is uniformly distributed in 1000-m radius of the BS. The number of groups of MTCDs is $K=10$. The other configuration parameters are $P_{k}^{\max }=14 \mathrm{dBm}, P_{\mathrm{G}}^{\max }=24 \mathrm{dBm}, T=1 \mathrm{~ms}, B_{k}=180 \mathrm{kHz}$, and $B_{G}=K B_{k}=1800 \mathrm{kHz}$

is built in MATLAB, it is difficult to compute the accurate convergence behavior of the optimal algorithm. The convergence behavior shown in the figure is an approximation). The cost of quick convergence of the proposed algorithm is less energy efficiency compared to the optimal algorithm, which implies that the complexity of the proposed algorithm is lower than the optimal algorithm, and is acceptable for the system.

Figure 5 demonstrates the influence on the total power efficiency of the MTCDs when the bandwidth of MTCG changes. From the formulation of MTCDs' data rate, it can be observed that the MTCG's transmission power

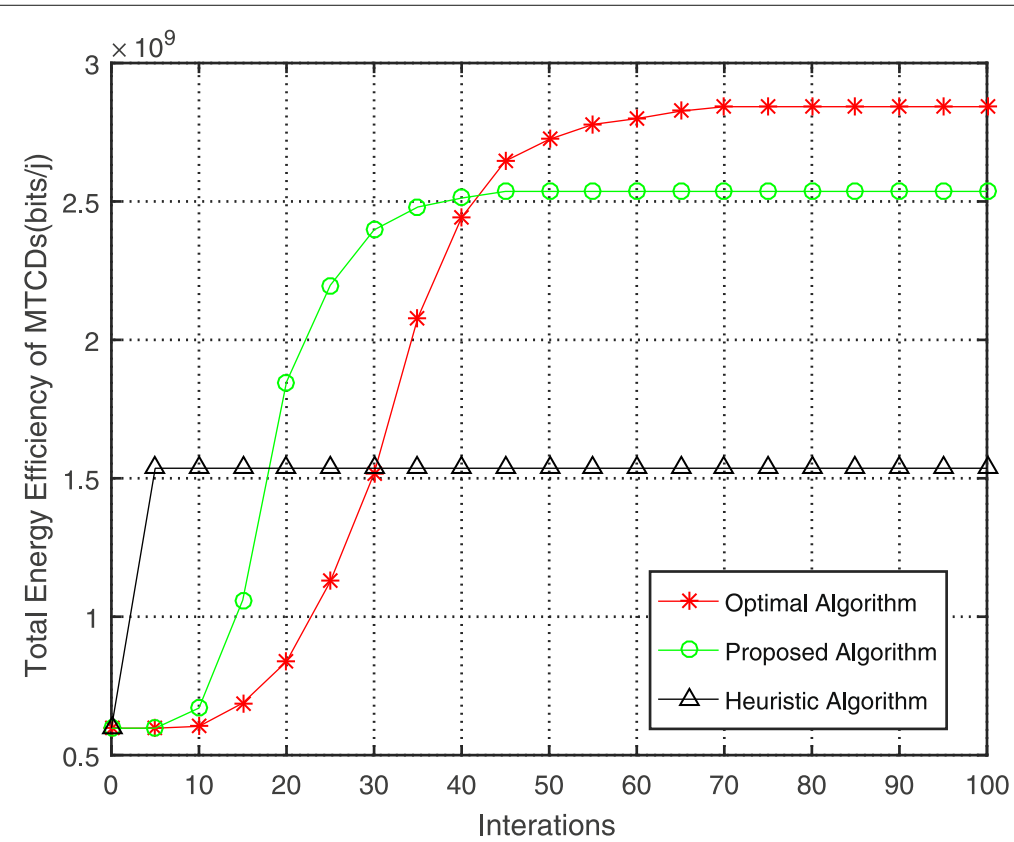

Fig. 4 Convergence behavior of the three algorithms. The MTCDs are uniformly distributed in 100-m radius of the MTCG, and the MTCG is uniformly distributed in 1000-m radius of the BS. The payload of each MTCD is $1500 \mathrm{bit} / \mathrm{TTI}$, and the self-interference of MTCG is $\Omega=1 \mathrm{~dB}$ 


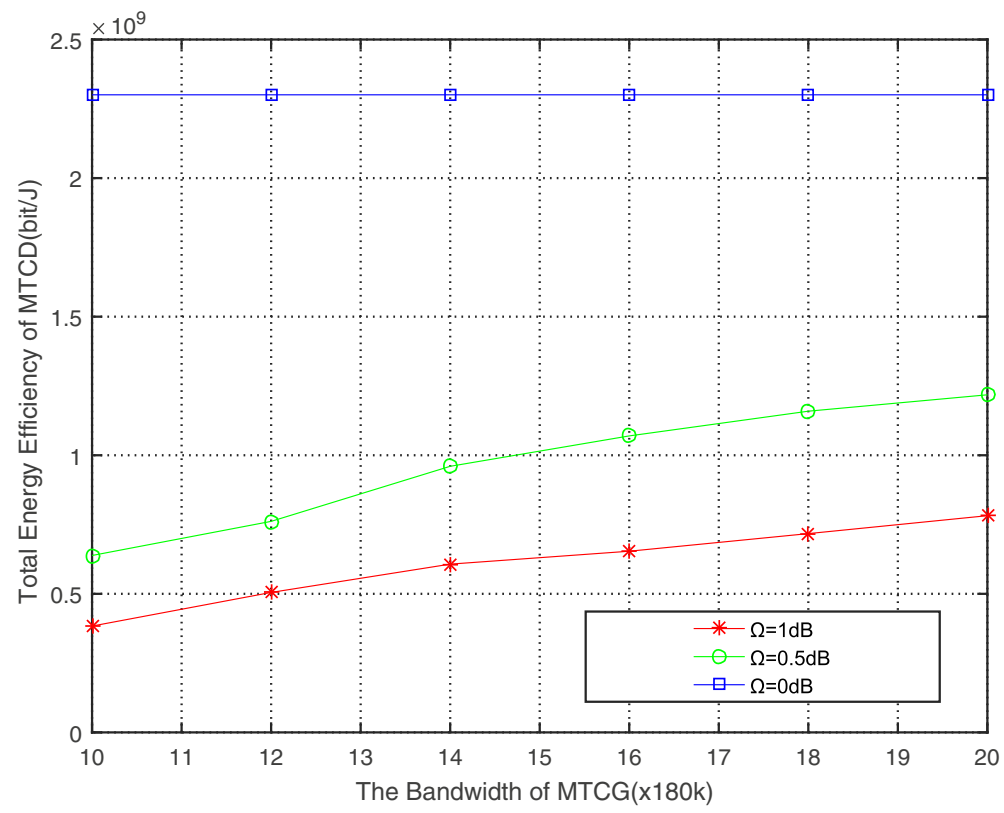

Fig. 5 The total energy efficiency with different MTCG's bandwidths. The MTCDs are uniformly distributed in 100-m radius of the MTCG, and the MTCG is uniformly distributed in 1000-m radius of the BS. The payload of each MTCD is $300 \mathrm{bit} / \mathrm{TT}$, and the number of groups of MTCDs is $K=10$

has nothing to do with MTCDs' data rate when there is no self-interference at MTCG. Thus, the change in the MTCG's bandwidth will not affect the devices' power efficiency when there is no self-interference at the MTCG. However, self-interference exists at the MTCG, and the powers of the MTCDs decrease with the increase in the bandwidth of the MTCG, increasing the total energy efficiency. This is due to the fact that the power of MTCG decreases when the MTCG's bandwidth increases, which leads to the decrease in the self-interference and then

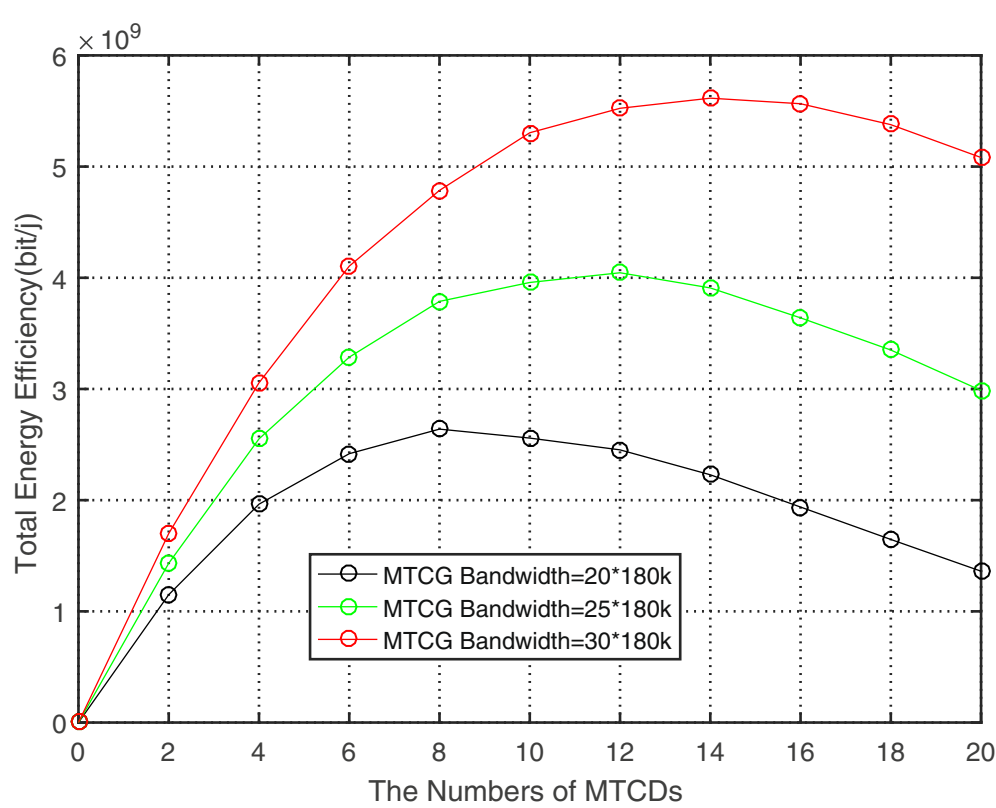

Fig. 6 The total energy efficiency with different numbers of MTCDs. The MTCDs are uniformly distributed in a 100-m radius of the MTCG, and the MTCG is uniformly distributed in a 1000-m radius of the BS. The payload of each MTCD is $1000 \mathrm{bit} / \mathrm{TT}$, and the self-interference of MTCG is $\Omega=1 \mathrm{~dB}$ 


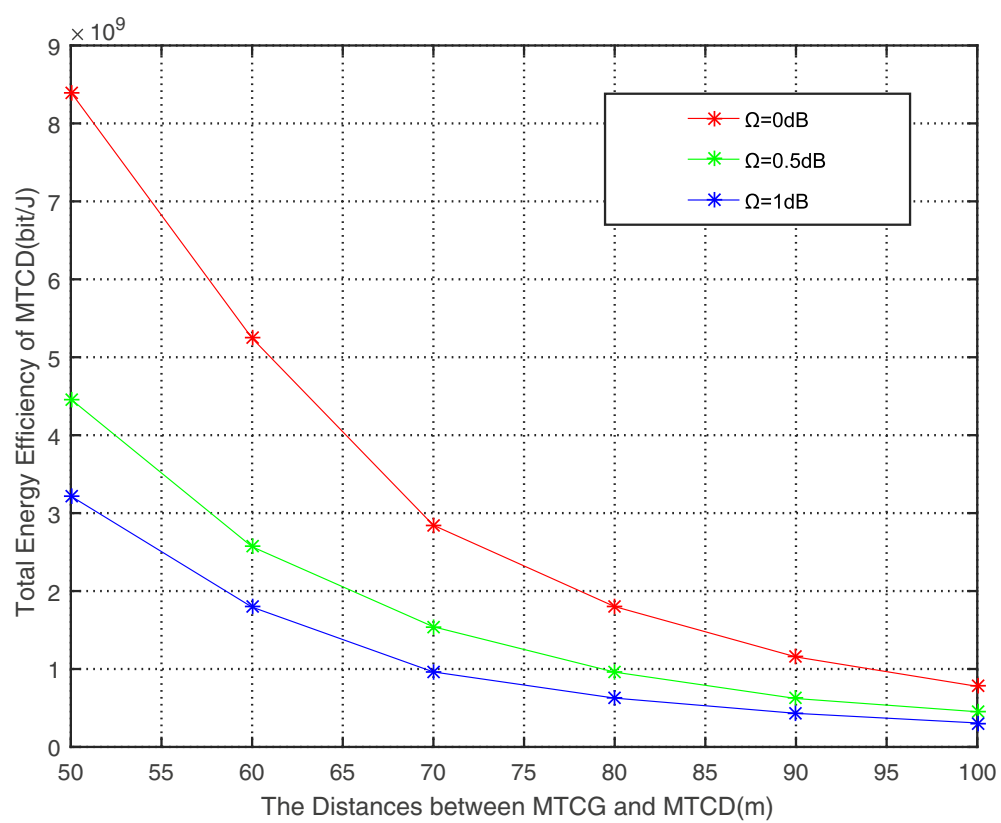

Fig. 7 The total energy efficiency with varying MTCD-to-MTCG distances. The payload of each MTCD is 1000 bit/TTI, the MTCG's bandwidth is $20 \times 180 \mathrm{kbit}$, and the number of groups of MTCDs is $K=10$

causes the MTCDs' power decrease. In addition, the total power efficiency increases with decrease in the selfinterference.

Figure 6 represents the total energy efficiency of MTCDs when the numbers of MTCDs change. It can be evidently seen that each MTCG bandwidth corresponds to the best number of MTCDs, which results in the total energy efficiency maximization. When the number of the MTCDs is less than the best number, the total energy efficiency increases with the increase in the number of MTCDs. The reason for this increase is that the MTCG's bandwidth is abundant to transmit the data. The MTCDs' transmit power increases slowly with the increase in the number of MTCDs. However, the data to be transmitted increases at a uniform rate, which is greater than the growth rate of MTCDs' power, thus increasing the total energy efficiency increases. When the number of MTCDs exceeds the best number, the MTCG's bandwidth becomes short for data transmission and the power consumption grows exponentially with the increasing number of MTCDs, e.g., the growth rate of power consumption is greater than the date rate increase speed that decreases the total energy efficiency. In summary, if it is required to increase the connected devices at MTCG, the MTCG bandwidth should also be increased to ensure the maximization of the total energy efficiency.

Figure 7 shows the relationship between the total power efficiency and the distances from the MTCDs to the MTCG. It can be observed from the figure that when the distances increase, the channel fading also increases due to the path loss that causes the increase of MTCDs' transmit power to ensure the transmit time requirements, then results in the total efficiency decreasing.

\section{Conclusions}

In this paper, a power allocation problem for M2M communication in single cell and multi-user with LTE-A cellular uplink is thoroughly investigated. In addition, an novela Lagrange multipliers-based power allocation algorithm is proposed that is acceptable in computational complexity to allocate the MTCDs' and the MTCG's power, with a group of MTCDs connected to the MTCG. Simulations are performed to analyze and compare the performance of the proposed Lagrange multipliers-based algorithm with the optimal algorithm and the heuristic algorithm. Furthermore, the influences on the total power efficiency of varying the information to be transmitted by the MTCDs are studied with and without the self-interference at the MTCG. Moreover, the relationship of the MTCDs' total energy efficiency and the bandwidth of the MTCG is analyzed. The simulation results demonstrate and validate that the proposed algorithm's performance is closer to the optimal algorithm and has an acceptable complexity.

\section{Abbreviations}

BS: Base station; FD: Full-duplex; H2H: Human-to-human communications; loT: Internet of Thing; LTE-A: Long Term Evolution-Advanced; M2M: Machine-to-machine communication; MTC: Machine-type communication; MTCD: Machine-type communication device; MTCG: Machine-type communication gateway; OFDMA: Orthogonal Frequency Division Multiple Access; PAN: Personal area network; PAPR: Peak-to-average-power ratio; QoS: 
Quality of services; RB: Resource block; SC-FDMA: Single Carrier Orthogonal Frequency Division Multiple Access; TTI: Transmission time intervals.

\section{Acknowledgements}

This work is supported by the National Natural Science Foundation of China (nos. 61771486 and 61571463).

\section{Funding}

This work is supported by the National Natural Science Foundation of China (nos. 61771486 and 61571463).

\section{Authors' contributions}

$\mathrm{NL}$ and $\mathrm{YH}$ conceived and designed the proposed scheme; they also performed the simulations. YH wrote the paper. CW, YC, and JX analyzed the simulation results. All of the authors participated in the project, and they read and approved the final manuscript.

\section{Competing interests}

The authors declare that they have no competing interests.

\section{Publisher's Note}

Springer Nature remains neutral with regard to jurisdictional claims in published maps and institutional affiliations.

Received: 13 March 2018 Accepted: 11 October 2018

Published online: 06 November 2018

\section{References}

1. P. Andres-Maldonado, P. Ameigeiras, J. Prados-Garzon, J. Navarro-Ortiz, J. M. Lopez-Soler, Narrowband IoT data transmission procedures for massive machine-type communications. IEEE Net. 31(6), 8-15 (2017)

2. G. Wu, S. Talwar, K. Johnsson, N. Himayat, K. D. Johnson, M2M: from mobile to embedded internet. IEEE Commun. Mag. 49(4), 36-43 (2011)

3. F. Ghavimi, H. H. Chen, M2M communications in 3GPP LTE/LTE-A networks: architectures, service requirements, challenges, and applications. IEEE Commun. Surv \& Tutor. 17(2), 525-549 (2015)

4. G. Wu, S. Talwar, K. Johnsson, N. Himayat, K. D. Johnson, M2M: from mobile to embedded internet. IEEE Commun. Mag. 49(4), 36-43 (2011)

5. A. Aijaz, A. H. Aghvami, in 2013 IEEE 77th Veh. Technol. Conf. On Radio Resource Allocation in LTE Networks with Machine-to-Machine Communications (VTC Spring, Dresden, 2013), pp. 1-5

6. A. Rajandekar, B. Sikdar, A survey of MAC layer issues and protocols for machine-to-machine communications. IEEE Internet Things J. 2(2), 175-186 (2015)

7. T. Taleb, A. Kunz, Machine type communications in 3GPP networks: potential, challenges, and solutions. IEEE Commun. Mag. 50(3), 178-184 (2012)

8. A. Ahmad, M. Assaad, in 2011 IEEE 22nd International Symposium on Personal, Indoor and Mobile Radio Communications. Power efficient resource allocation in uplink SC-FDMA systems, (Toronto, 2011), pp. 1351-1355

9. D. Feng, C. Jiang, G. Lim, L. J. Cimini, G. Feng, G. Y. Li, A survey of energy-efficient wireless communications. IEEE Commun. Surv.\& Tutor. 15(1), 167-178 (2013)

10. F. Ghavimi, Y. W. Lu, H. H. Chen, Uplink scheduling and power allocation for M2M communications in SC-FDMA-based LTE-A networks with QoS guarantees. IEEE Trans. Veh. Technol. 66(7), 6160-6170 (2017)

11. G. Zhang, A. Li, K. Yang, L. Zhao, D. Cheng, Optimal power control for delay-constraint machine type communications over cellular uplinks. IEEE Commun. Lett. 20(6), 1168-1171 (2016)

12. G. Zhang, A. Li, K. Yang, L. Zhao, Y. Du, D. Cheng, Energy-efficient power and time-slot allocation for cellular-enabled machine type communications. IEEE Commun. Lett. 20(2), 368-371 (2016)

13. A. Aijaz, M. Tshangini, M. R. Nakhai, X. Chu, A. H. Aghvami, Energy-efficient uplink resource allocation in LTE networks with $\mathrm{M} 2 \mathrm{M} / \mathrm{H} 2 \mathrm{H}$ co-existence under statistical QoS guarantees. IEEE Trans. Commun. 62(7), 2353-2365 (2014)

14. L. Rongkuan, P. Popovskiy, W. Gang, in 2015 10th International Conference on Communications and Networking in China (ChinaCom). Adaptive link selection and power allocation buffer-aided relay networks with multiple sources, (Shanghai, 2015), pp. 312-316
15. J. Tang, D. K. C. So, N. Zhao, A. Shojaeifard, K. K. Wong, Energy efficiency optimization with SWIPT in MIMO broadcast channels for Internet of Things. IEEE Internet of Things J.

16. J. Tang, D. K. C. So, E. Alsusa, K. A. Hamdi, A. Shojaeifard, Resource allocation for energy efficiency optimization in heterogeneous networks. IEEE Sel, J., Areas Commun. 33(10), 2104-2117 (2015)

17. V. Rodoplu, T. H. Meng, Bits-per-joule capacity of energy-limited wireless networks. IEEE Trans. Wirel. Commun. 6(3), 857-865 (2007)

18. S. Kim, B. G. Lee, D. Park, Energy-per-bit minimized radio resource allocation in heterogeneous networks. IEEE Trans. Wirel. Commun. 13(4), 1862-1873 (2014)

19. P. P. Mariio, in Optimization of Computer Networks: Modeling and Algorithms: A Hands-On Approach. Appendix B: mathematical optimization basics, vol. 1 (Wiley Telecom, 2015), p. 420. https://doi.org/ 10.1002/9781119114840app2

20. G. Zheng, Joint Beamforming Optimization and power control for full-duplex MIMO two-way relay channel. IEEE Trans. Signal Proc. 63(3), 555-566 (2015)

21. G. Rigazzi, N. K. Pratas, P. Popovski, R. Fantacci, in 2015 IEEE International Conference on Communications (ICC). Aggregation and trunking of M2M traffic via D2D connections, (London, 2015), pp. 2973-2978

22. I. C. Wong, O. Oteri, W. Mccoy, Optimal resources allocation inuplink SC-FDMA systems. IEEE Trans. Wirel. Commun. 8(5), 2161-2165 (2009)

23. R. O'Donnell, Prolog to a unified approach to the performance analysis of digital communication over generalized fading channels. Proc. IEEE. 86(9), 1858-1859 (1998)

24. Lecture Slides on Class EE364b of Stanford Univ. S. Boyd, Ellipsoid Method. https://www.stanford.edu/class/ee364b/lectures.html. Accessed 16 Dec 2017

25. M. Tao, Y. C. Liang, F. Zhang, Resource allocation for delay differentiated traffic in multiuser OFDM systems. IEEE Trans. Wirel. Commun. 7(6), 2190-2201 (2008)

\section{Submit your manuscript to a SpringerOpen ${ }^{\circ}$ journal and benefit from:}

- Convenient online submission

- Rigorous peer review

- Open access: articles freely available online

- High visibility within the field

- Retaining the copyright to your article

Submit your next manuscript at $\mathbf{s p r i n g e r o p e n . c o m ~}$ 